\title{
STAT3 mutation impacts biological and clinical features of T-LGL leukemia
}

\author{
Antonella Teramo ${ }^{1,2, *}$, Gregorio Barilà ${ }^{1,2, *}$, Giulia Calabretto ${ }^{1,2}$, Chiara Ercolinn ${ }^{1,2}$, \\ Thierry Lamy ${ }^{3}$, Aline Moignet ${ }^{3}$, Mikael Roussel ${ }^{4}$, Cédric Pastoret ${ }^{4}$, Matteo Leoncin ${ }^{1}$, \\ Cristina Gattazzo ${ }^{1,2}$, Anna Cabrelle ${ }^{2}$, Elisa Boscaro ${ }^{1}$, Sara Teolato ${ }^{1}$, Elisa Pagnin ${ }^{1}$, \\ Tamara Berno ${ }^{1}$, Elena De March1, Monica Facco ${ }^{1,2}$, Francesco Piazza ${ }^{1,2}$, Livio \\ Trentin $^{1,2}$, Gianpietro Semenzato ${ }^{1,2}$ and Renato Zambello ${ }^{1,2}$ \\ ${ }^{1}$ Padua University School of Medicine, Department of Medicine, Hematology and Clinical Immunology Branch, Padua, Italy \\ ${ }^{2}$ Venetian Institute of Molecular Medicine (VIMM), Padua, Italy \\ ${ }^{3}$ Department of Clinical Hematology, University Hospital of Rennes, Rennes, France \\ ${ }^{4}$ Biology Department, University Hospital of Rennes, Rennes, France \\ *These authors have contributed equally to this work \\ Correspondence to: Renato Zambello, email: r.zambello@unipd.it \\ Keywords: large granular lymphocyte leukemia, STAT3 mutation, immunophenotype, neutropenia, fas ligand \\ Received: January 20, $2017 \quad$ Accepted: May 22, $2017 \quad$ Published: June 27, 2017 \\ Copyright: Teramo et al.This is an open-access article distributed under the terms of the Creative Commons Attribution License \\ 3.0 (CC BY 3.0), which permits unrestricted use, distribution, and reproduction in any medium, provided the original author and \\ source are credited.
}

\section{ABSTRACT}

STAT3 mutations have been described in $30-40 \%$ of T-large granular lymphocyte (T-LGL) leukemia patients, leading to STAT3 pathway activation. Considering the heterogeneity of the disease and the several immunophenotypes that LGL clone may express, the aim of this work was to evaluate whether STAT3 mutations might be associated with a distinctive LGL immunophenotype and/or might be indicative for specific clinical features.

Our series of cases included a pilot cohort of 101 T-LGL leukemia patients ( 68 CD8+/CD4- and 33 CD4+/CD8 \pm ) from Padua Hematology Unit (Italy) and a validation cohort of additional 20 patients from Rennes Hematology Unit (France).

Our results indicate that i) CD8+ T-LGL leukemia patients with CD16+/CD56immunophenotype identify a subset of patients characterized by the presence of STAT3 mutations and neutropenia, ii) CD4+/CD8 \pm T-LGL leukemia are devoid of STAT3 mutations but characterized by STAT5b mutations, and iii) a correlation exists between STAT3 activation and presence of Fas ligand, this molecule resulting highly expressed in CD8+/CD16+/CD56- patients. Experiments with stimulation and inhibition of STAT3 phosphorylation confirmed this relationship. In conclusion, our data show that T-LGL leukemia with specific molecular and phenotypic patterns is associated with discrete clinical features contributing to get insights into molecular bases accounting for the development of Fas ligand-mediated neutropenia.

\section{INTRODUCTION}

T-cell large granular lymphocyte (T-LGL) leukemia is a rare chronic lymphoproliferative disorder characterized by the clonal expansion of CD3+ Large Granular Lymphocytes (LGL) [1-3]. T-LGLs typically exhibit a post-thymic mature effector memory phenotype (CD3+/CD8+/CD57+/CD45RA+/ CD62L-) and variable expression of CD16, CD56 and NK receptors, namely Killer Immunoglobulin-like Receptor (KIR) and CD94/ NKG2, indicating that these cells represent late stage fully differentiated cytotoxic T-lymphocytes $[4,5]$. In addition 
to the most common CD8+ T-LGL leukemia, less frequent LGL proliferations with $\mathrm{CD} 4+/ \mathrm{CD}^{-/+\operatorname{dim}}$ phenotype (CD4+ T-LGL leukemia) exist, which are characterized by a typical V $\beta 13.1$ expression, frequent association with secondary neoplasia and a pathogenetic relationship with CMV infection [6].

The etiology of T-LGL leukemia is still unknown. Several reports support the hypothesis that chronic antigenic stimulation induces monoclonal LGL proliferation persisting through defective activation induced cell death (AICD). Several pathways are constitutively activated in T-LGL leukemia leading to resistance to apoptosis, such as MAPK/ERK, PI3K/AKT, NF- $\kappa$ B pathways, sphingolipid rheostat and JAK/STAT pathway [7]. Epling-Burnette et al [8] first demonstrated constitutive STAT3 activation with Mcl1 upregulation in T-LGL leukemia. We previously identified both extrinsic (IL-6 trans-signaling) and intrinsic mechanisms (SOCS3 downregulation) contributing to constitutive STAT3 tyrosine phosphorylation [9]. Moreover, somatic STAT3 and $S T A T 5 b$ mutations determining constitutive activation have been reported, the former detected in a proportion of approximately $40 \%$ of patients $[10,11]$ and the latter being associated to aggressive LGL disorders [12] and, as recently reported, to CD4+ T-LGL leukemia patients (6 out of 11 cases) [13]. Some authors reported that STAT3 genetic lesions are associated with neutropenia $[10,14]$, although this correlation has not yet been specifically evaluated also in consideration that the pathogenesis of neutropenia is likely to be multifactorial, comprising both humoral and cytotoxic mechanisms [15]. Since normal neutrophil survival is partly regulated by the Fas-Fas ligand apoptotic system, it is suggested that LGL leukemia neutropenia might be mediated by deregulated expression of Fas ligand. Consistently, high levels of circulating Fas ligand have been detected in T-LGL leukemia serum, likely triggering neutrophil apoptosis through the production of secreted Fas ligand [16].

Taking into account the heterogeneity of the disease and the several immunophenotypes that may characterize LGL clone, the aim of this work was to evaluate whether STAT3 mutations might be associated with a distinctive LGL immunophenotype and/or indicative for symptomatic disease in an initial cohort of 101 patients affected by T-LGL leukemia. Our results demonstrate that, in CD8+ T-LGL leukemia, the CD16+/ CD56- immunophenotype is associated with STAT3 mutations, identifying a more symptomatic and treatment requiring disease. The predictive value of CD8+/CD16+/ CD56- immunophenotype to identify STAT3 mutated and neutropenic patients was also confirmed in a validation cohort of 20 patients from Rennes University (France). The evidence that CD8+/CD16+/CD56- patients were characterized by higher level of Fas ligand, as a consequence of their higher STAT3 phosphorylation, offers a mechanistic explanation for the correlation between STAT3 activation and neutropenia.

\section{RESULTS}

\section{STAT3 mutations}

In the pilot cohort, we observed 38 patients out of 101 analyzed (37.6\%) carrying STAT3 mutations, 36 patients by Sanger sequencing and 2 more cases by ARMS-PCR (amplification refractory mutation system, an assay revealing Y640F and D661Y undetectable by Sanger sequencing if present in less than $25 \%$ of cells). All samples were evaluated at diagnosis. STAT3 mutations were always found in leukemic LGLs and not in the remaining non-leukemic peripheral blood mononuclear cells (PBMCs) (data not shown). In two cases bone marrow cells were available and the same mutation was identified in both peripheral blood and bone marrow. The distribution of mutations was as follows: 24 cases presented Y640F (63.2\%), 9 cases D661Y (23.7\%), one case D661V (2.6\%), one case N647I (2.6\%), and 3 cases presented mutations not yet described in T-LGL leukemia. These latter were the following: one case with a point mutation, K658R, together with an in-frame insertion, I659_M660insL (2.6\%), a second case with an in-frame deletion/insertion, A662_N663delinsH (2.6\%), and a third case with an in-frame insertion, G656_Y657insY (Figure 1). Surprisingly, a significant correlation was demonstrated between the presence of STAT3 mutations and female gender $\left(\chi^{2}=3.91, P<0.05\right.$; Table 1$)$.

\section{Clinical characteristics in T-LGL leukemia patients}

Among the 101 patients (50 males, 51 females) of the pilot cohort, neutropenia (absolute neutrophil count, ANC $<1,500 \mathrm{~mm}^{3}$ ) was documented in 39 patients (38.6\%), with $17(16.8 \%)$ presenting severe neutropenia $\left(\right.$ ANC $<500 \mathrm{~mm}^{3}$ ) (Table 1). Thirty-four out of 39 neutropenic patients were STAT3 mutated (87.2\%), all the patients characterized by severe neutropenia being included among the group of mutated cases. Only 5 patients were neutropenic and did not display STAT3 mutations. This correlation between the presence of STAT3 mutations and neutropenia was demonstrated highly statistically significant $\left(\chi^{2}=66.5, P<0.0001\right.$; Table 1$)$. No correlation between neutrophils' count and the kind of STAT3 mutation or percentage of mutated clone was found. In fact, a patient mutated only by ARMS-PCR had severe neutropenia, vice-versa a patient homozygous for mutation has never experimented severe neutropenia. Similarly, no correlation between neutrophils' count and LGL percentage or LGL absolute number were observed (data not shown). 
Rheumatoid Arthritis (RA) was present in three patients $(3 \%)$, all mutated and neutropenic. The frequency of RA was lower than previously reported in the literature $[17,18]$, this can be explained because in our cohort only symptomatic patients who required specific treatment were considered affected by RA; a Reumathoid factor or antiCytrulline antibody positivity was not considered sufficient to make diagnosis of RA. Anyway, the association between RA and STAT3 mutation described in previous study [10] was confirmed. The association with different autoimmune disorders (i.e., autoimmune hemolytic anemia, immune thrombocytopenia, Hashimoto Thyroiditis, Systemic Erythematosus Lupus and Vitiligo) was observed in 23 patients $(22.8 \%)$, mostly mutated patients $(16 / 23,69.6 \%$; $\left.\chi^{2}=12.9, P<0.0001\right)$. Differently, concomitant second neoplasia (benign or malignant), affecting 28 out of 101 patients $(27.7 \%)$, was not statistically associated with wild type or mutated patients $\left(\chi^{2}=1.35, P=0.245\right.$; Table 1$)$.

According to our policy to treat patients when symptomatic for neutropenia and not according to neutrophils number, at the time of the study only 13 patients required treatment, 12 out of 13 were STAT3 mutated $\left(92.3 \% ; \chi^{2}=19.0, P<0.0001\right.$; Table 1$)$. The first perspective trial evaluating the efficacy of immunosuppressive therapy in LGL leukemia evidenced the relationship between the presence of Y640F STAT3 mutation and response to MTX [19]. In our cohort, 6 patients were Y640F mutated and received MTX therapy obtaining an overall response rate of $67 \%$, with one complete response. Even if in a low number of cases, this result supports the previous data described by Loughran et al [19].

\section{Immunophenotypic characterization of patients T-LGLs}

By flow analysis, we observed that 68 out of 101 patients $(67.3 \%)$ were characterized by classical CD3+/CD8+/CD4- expression (CD8+ T-LGL leukemia), while the remaining 33 patients $(32.7 \%)$ were distributed among CD3+/CD4+/CD8+dim $(n=23,22.8 \%)$ and CD3+/ CD4+/CD8- $(n=10,9.9 \%)$ phenotypes. From now on we report these two latter subsets together under the definition of CD4+ T-LGL leukemia. According to the expression of the NK cell markers CD16, CD56 and CD57, several possible immunophenotype combinations were demonstrated in both CD8+ and CD4+ T-LGL leukemia. More specifically, according to positivity and negativity of the three markers listed above, CD8+ T-LGL leukemia displayed five different immunophenotypes, while CD4+ T-LGL leukemia exhibited only two dominant phenotypes (data not shown).

All STAT3 mutated $(n=38)$ and almost all neutropenic (38 out of 39) patients belonged to CD8+ T-LGL leukemia $(n=68)$, while among CD4+ T-LGL leukemia $(n=33)$ only one neutropenic patient (1 out of $33,3 \%$ ) was found, this last characterized by mild neutropenia (ANC: $1,470 \mathrm{~mm}^{3}$ ) over 9 years mean follow up.

Interestingly, all the STAT3 mutated samples were mostly characterized by CD16+/CD56-/CD57+ (33 out of $38,86.8 \%$ ), followed by CD16+/CD56-/CD57phenotypes (4 out of 38, 10.5\%; Figure 2, left pie chart). At variance, in CD8+ T-LGL leukemia wild type group $(n=30)$, these two immunophenotypes were barely
24 cases $(63.2 \%)$

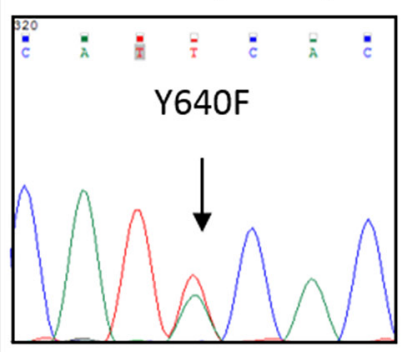

1 case $(2.6 \%)$

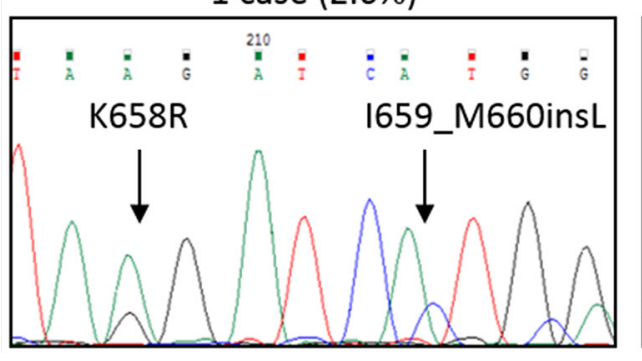

9 cases $(23.7 \%)$

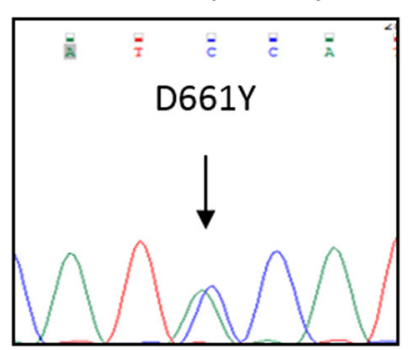

1 case $(2.6 \%)$

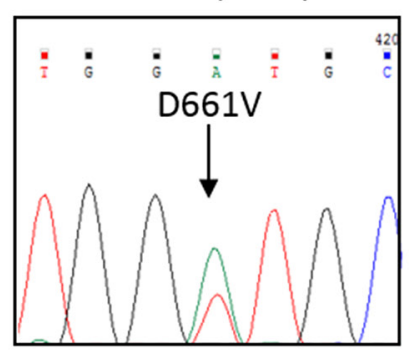

1 case $(2.6 \%)$

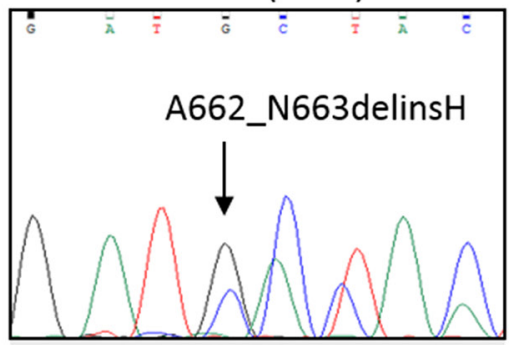

1 case $(2.6 \%)$

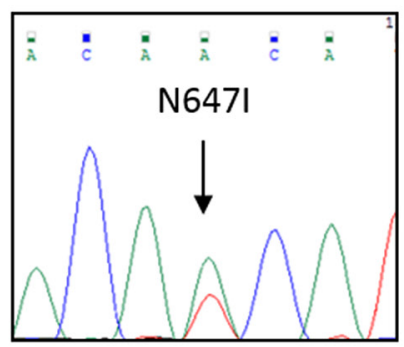

1 case $(2.6 \%)$

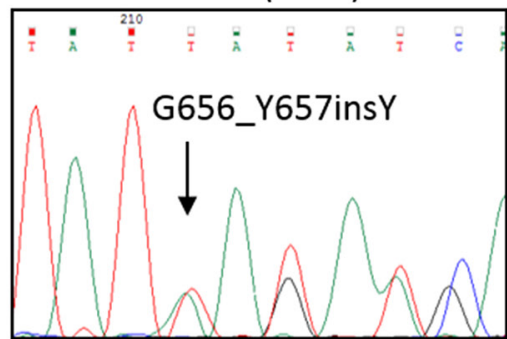

Figure 1: Representative Sanger sequences for each STAT3 mutation found. By Sanger sequencing, STAT3 mutations were observed in 36 out of 101 T-LGL leukemia patients. Two more cases were found by ARMS-PCR. Upper each graph the cases and their incidence (\%) among STAT3 mutated patients $(n=38)$ are indicated. Y640F and D661Y accounted for the most frequent mutations found. 
Table 1: Evaluation of $S T A T 3$ mutations incidence in T-LGL leukemia patients according to clinical characteristics

\begin{tabular}{|c|c|c|c|c|c|}
\hline Variable & $\begin{array}{c}\text { T-LGL } \\
\text { leukemia } \\
\text { patients } \\
n=101\end{array}$ & $\begin{array}{c}\text { Patients } \\
\text { with } S T A T 3 \\
\text { mutation } \\
\quad n=38\end{array}$ & $\begin{array}{c}\text { Patients } \\
\text { without } S T A T 3 \\
\text { mutation } \\
n=63\end{array}$ & $\boldsymbol{P}$ & STAT3 mutations \\
\hline Gender & & & & 0.048 & \\
\hline Males & $50(50 \%)$ & $14(37 \%)$ & $36(57 \%)$ & & $\begin{array}{l}8 \text { Y640F, } 2 \text { D661Y, } \\
1 \text { D661V, } 1 \text { N647I, } \\
1 \text { G656_Y657insY, } \\
1 \text { K658R and I659_- } \\
\text { M660insL }\end{array}$ \\
\hline Females & $51(50 \%)$ & $24(63 \%)$ & $27(43 \%)$ & & $\begin{array}{l}16 \text { Y640F, } 7 \text { D661Y, } \\
1 \text { A662_N663delinsH }\end{array}$ \\
\hline $\begin{array}{l}\text { Neutropenia } \\
(\text { ANC < 1,500) }\end{array}$ & $39(39 \%)$ & $34(89 \%)$ & $5(8 \%)$ & 0.0001 & $\begin{array}{l}21 \text { Y640F, } 8 \text { D661Y, } \\
1 \text { D661 V, 1 N647I, } \\
1 \text { G656_Y657insY, } \\
1 \text { K658R and I659- } \\
\text { M660insL, } 1 \text { A662_- } \\
\text { N663delinsH }\end{array}$ \\
\hline $\begin{array}{l}\text { Severe neutropenia } \\
(\mathrm{ANC}<\mathbf{5 0 0})\end{array}$ & $17(17 \%)$ & $17(45 \%)$ & 0 & 0.0001 & $\begin{array}{l}10 \text { Y640F, } 4 \text { D661Y, } 1 \\
\text { N647I, } 1 \text { K658R and } \\
\text { I659_M660insL, 1 } \\
\text { A662_N663delinsH }\end{array}$ \\
\hline Rheumatoid arthritis & $3(3 \%)$ & $3(8 \%)$ & 0 & 0.024 & 2 Y640F, 1 D661Y \\
\hline $\begin{array}{l}\text { Other autoimmune } \\
\text { diseases }\end{array}$ & $23(23 \%)$ & $16(42 \%)$ & $7(11 \%)$ & 0.0001 & $\begin{array}{l}11 \text { Y640F, } 4 \text { D661Y, } \\
1 \text { K658R and I659_- } \\
\text { M660insL }\end{array}$ \\
\hline Associated neoplasia & $28(28 \%)$ & $8(21 \%)$ & $20(32 \%)$ & 0.245 & $\begin{array}{l}5 \text { Y640F, } 2 \text { D661Y, } \\
1 \text { K658R and I659- } \\
\text { M660insL }\end{array}$ \\
\hline Treatment & $13(13 \%)$ & $12(32 \%)$ & $1(2 \%)$ & 0.0001 & $\begin{array}{l}8 \text { Y640F, } 3 \text { D661Y, } \\
1 \text { G656_Y657insY }\end{array}$ \\
\hline
\end{tabular}

ANC, absolute neutrophil count.

detected (4 out of $30,13.3 \%$ ) or absent, respectively (Figure 2, right pie chart). Only one patient with mutated STAT3 had a different markers expression, being CD16-/ CD56-/CD57+ (1 out of 38, 2.6\%; Figure 2, left pie chart). In conclusion, the two main immunophenotypes of STAT3 mutated group were characterized by CD16 positivity and CD56 negativity, defining an immunophenotype signature CD16+/CD56- $(n=41)$ strongly and significantly associated with the presence of STAT3 mutations (37 out of $\left.41,90.2 \% ; \chi^{2}=49.5, P<0.0001\right)$. Moreover, this immunophenotypic signature identified even almost all neutropenic patients (37 out of $41,90.2 \% ; \chi^{2}=49.5, P$ $<0.0001$; Figure 3A), also including those 4 cases CD8+ T-LGL leukemia with neutropenia that had wild type STAT3. In more detail, among the 41 patients with CD16+/ CD56- immunophenotype, 33 were both mutated and neutropenic, 4 were STAT3 mutated without neutropenia and other 4 were neutropenic with wild type STAT3.

Within the 3 other immunophenotypes of CD8+ T-LGL leukemia any other case did not show neutropenia but one $(1 / 27,3.7 \%$; Figure $3 \mathrm{~A})$, that was the only STAT3 mutated case out of the most common immunophenotypic signature (CD16+/CD56-) and it belonged to CD16-/ CD56-/CD57+ subset.

All the data are listed in Table 2. As indicated, CD16+/CD56- subgroup showed the ANC value significantly lower than that observed in the remaining CD8+ groups and CD4+ T-LGL leukemia patients (mean $\mathrm{ANC} \pm \mathrm{SEM}: 795.46 \pm 80.03 \mathrm{~mm}^{3}, 2,855.11 \pm 224.54 \mathrm{~mm}^{3}$; $2,635.76 \pm 193.81 \mathrm{~mm}^{3}$, respectively, $P<0.05$; Figure $3 \mathrm{~B}$ ).

Vbeta analysis was performed on the entire cohort of patients and no specific association was found between 
the presence of STAT3 mutations, neutropenia and Vbeta usage. On the contrary, among CD4+ LGL leukemia patients a high frequency of Vbeta 13.1 was shown (9/29, $31 \%$ ) as previously reported [6].

\section{CD8+ T-LGL leukemia immunophenotypes

$\begin{array}{cc}\text { STAT3 mutated } & \text { STAT3 wild type } \\ \text { patients }(n=38) & \text { patients }(n=30)\end{array}$
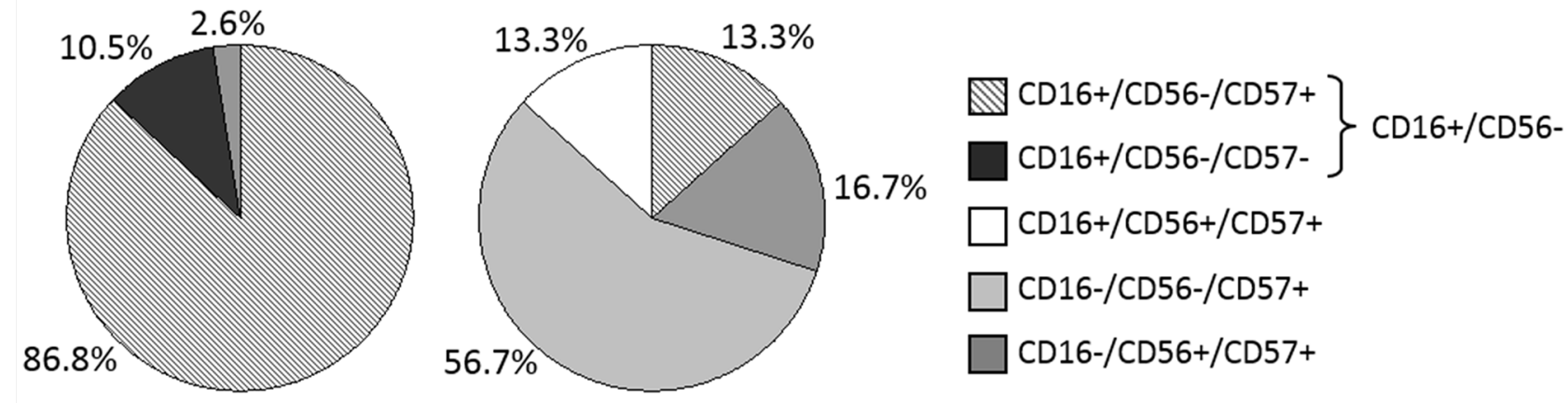

Figure 2: Immunophenotype distribution analysis evaluated in $S T A T 3$ mutated patients as compared with $S T A T 3$ wild type patients within CD8+ T-LGL leukemia. The graphs represent the incidence (\%) of each LGL immunophenotype in the group of patients with STAT3 mutations (pie chart on the left) and in the group wild type for STAT3 gene (pie chart on the right). Mutated patients mostly belong to CD16+/CD56- phenotype (37/41, 90.2\%), representing only $13.3 \%$ (4/30) of wild type patients' group.

\section{A Neutropenic patients (\%)}

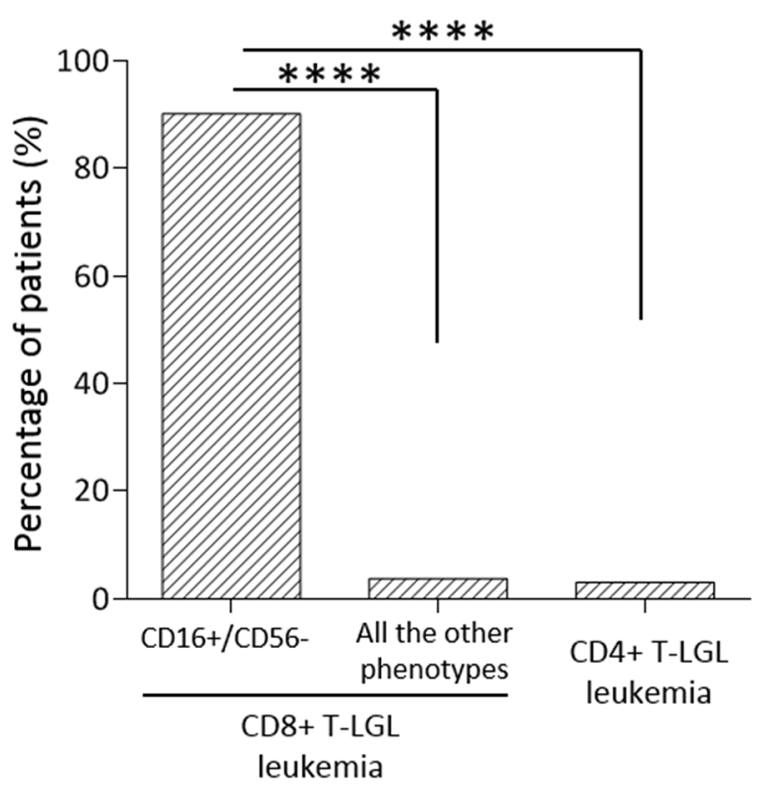

B Average of ANC $\mathrm{mm}^{3}$

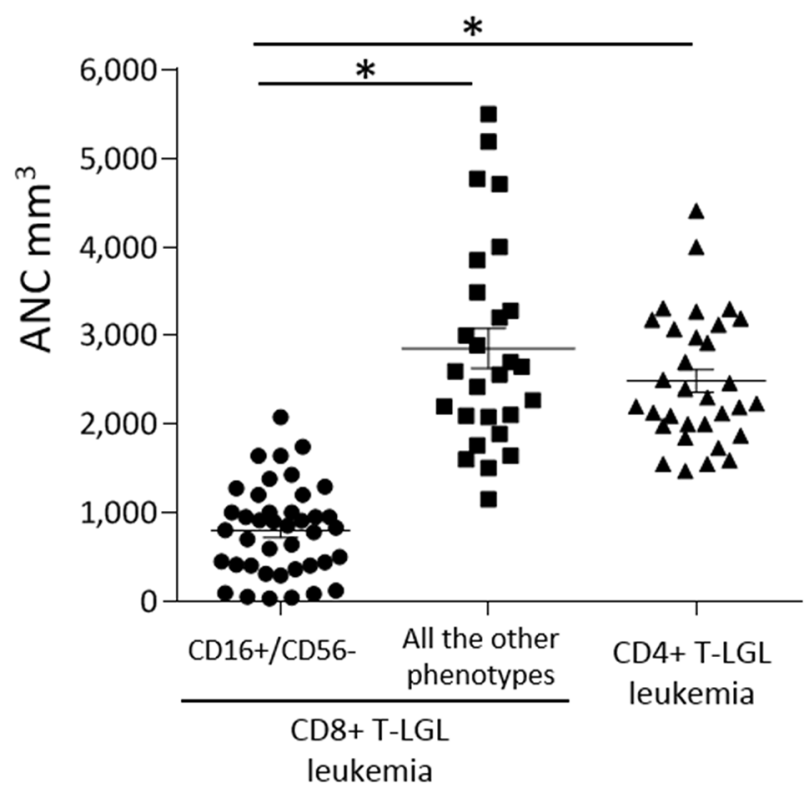

Figure 3: Neutropenia evaluation in the patients subdivided according to their immunophenotypes. (A) Histogram of the percentages of patients with neutropenia $\left(\mathrm{ANC}<1,500 \mathrm{~mm}^{3}\right)$. Neutropenia incidence resulted $90.2 \%$ in CD16+/CD56- subset $(n=41)$, $3.7 \%$ in the other immunophenotypic subsets of CD8+ T-LGL leukemia $(n=27)$ and $3 \%$ in CD4+ T-LGL leukemia $(n=33)$, the difference is highly statistically significant $\left(* * * * P<0.0001, \chi^{2}=49.5\right.$ and $\chi^{2}=55.7$, respectively, using $\chi^{2}$ test). (B) Dot plot indicating ANC level of each patient. The mean of ANC \pm SEM in CD16+/CD56- subset $\left(795.46 \pm 80.03 \mathrm{~mm}^{3}\right)$ is lower than in the other immunophenotypic subsets of CD8+ T-LGL leukemia $\left(2,855.11 \pm 224.54 \mathrm{~mm}^{3}\right)$ and in CD4+ T-LGL leukemia $\left(2,635.76 \pm 193.81 \mathrm{~mm}^{3} ;{ }^{*} P<0.05\right.$, using one-way Anova and Tukey's multiple comparison test). 
Table 2: Comparison of biological and clinical variables within CD8+ T-LGL leukemia between CD16+/CD56- and the other immunophenotypes

\begin{tabular}{|c|c|c|c|c|}
\hline Variable & $\begin{array}{c}\text { CD8+ T-LGL } \\
\text { leukemia patients } \\
n=68\end{array}$ & $\begin{array}{c}\text { CD16+/CD56- } \\
\text { patients } \\
n=41\end{array}$ & $\begin{array}{c}\text { Patients with other } \\
\text { immunophenotypes } \\
n=27\end{array}$ & $P$ \\
\hline Gender & & & & 0.033 \\
\hline Males & $32(47 \%)$ & $15(37 \%)$ & $17(63 \%)$ & \\
\hline Females & $36(53 \%)$ & $26(63 \%)$ & $10(37 \%)$ & \\
\hline STAT3 mutation & $38(56 \%)$ & $37(90 \%)$ & $1(4 \%)$ & 0.0001 \\
\hline Neutropenia $(A N C<1,500)$ & $38(56 \%)$ & $37(90 \%)$ & $1(4 \%)$ & 0.0001 \\
\hline $\begin{array}{l}\text { Severe neutropenia (ANC } \\
<500)\end{array}$ & $17(25 \%)$ & $17(41 \%)$ & 0 & 0.0001 \\
\hline Rheumatoid arthritis & $3(4 \%)$ & $3(7 \%)$ & 0 & 0.151 \\
\hline Other autoimmune diseases & $20(29 \%)$ & $16(39 \%)$ & $4(15 \%)$ & 0.032 \\
\hline Associated neoplasia & $16(24 \%)$ & $8(20 \%)$ & $8(30 \%)$ & 0.336 \\
\hline Treatment & $13(19 \%)$ & $12(29 \%)$ & $1(4 \%)$ & 0.009 \\
\hline
\end{tabular}

ANC, absolute neutrophil count.

\section{Validation cohort}

To confirm the correlation among immunophenotype, presence of STAT3 mutations and neutropenia observed in the pilot study group, we evaluated an independent cohort of 20 patients from University Hospital Hematology Unit of Rennes, France. Specifically, we took into account a group of patients affected by CD8+ T-LGL leukemia and characterized by the immunophenotype of interest, CD16+/CD56- (Table 3). All samples were evaluated at diagnosis. In this cohort, 17 out of 20 patients were STAT3 mutated and neutropenic. The remaining 3 patients were wild type for STAT3 and only one was neutropenic. In this different cohort of patients, the presence of STAT3 mutations and neutropenia resulted with an incidence of $85 \%$ and $90 \%$, respectively, confirming the findings of the pilot cohort.

\section{STAT3 phosphorylation}

By western blot analysis, we showed that high STAT3 tyrosine phosphorylation was observed in LGL samples obtained by CD8+ T-LGL leukemia patients belonging to CD16+/CD56- subgroup, either they were mutated or wild type, either neutropenic or not. The average values, evaluated by densitometry on 26 samples, resulted statistically higher in these patients as compared with all the other immunophenotypic groups, evaluated on 23 samples (P-STAT3/STAT3, median \pm SEM: $1.89 \pm$ 0.78 and $0.49 \pm 0.10$, respectively, $P<0.01$; Figure $4 \mathrm{~A}$ ).
A

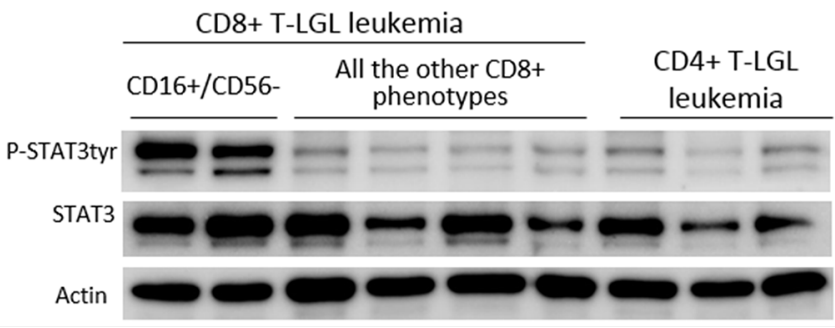

B

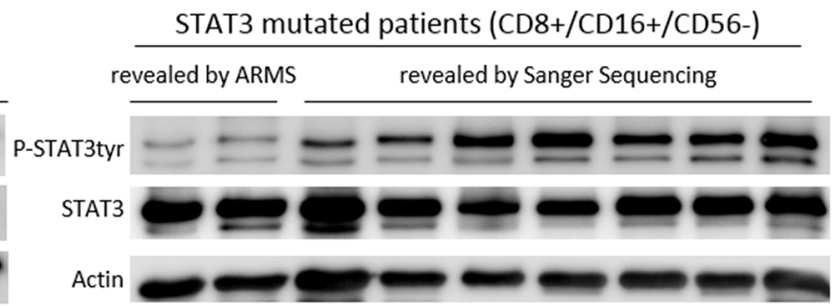

Figure 4: Western blot analysis of LGLs' extracts for phosphorylated STAT3, total STAT3 and Actin. (A) Representative cases for the patients subdivided according to their different immunophenotypes are reported. (B) Representative cases for STAT3 mutated patients subdivided into samples where mutation was revealed only by ARMS-PCR (mutated clone $<25 \%$ of the entire clone) and those with mutations revealed by Sanger Sequencing. Actin expression is shown as gel loading control. 
Table 3: Comparison of biological and clinical variables of the Italian and French cohorts of CD16+/CD56- CD8+ T-LGL leukemia patients

\begin{tabular}{lcc}
\hline Variable & $\begin{array}{c}\text { CD16+/CD56- CD8+ T-LGL } \\
\text { leukemia patients } \\
\text { (Padua, Italy) } \\
\boldsymbol{n}=\mathbf{4 1}\end{array}$ & $\begin{array}{c}\text { CD16+/CD56- CD8+ T-LGL } \\
\text { leukemia patients } \\
\text { (Rennes, France) } \\
\mathbf{2 0}\end{array}$ \\
\hline Gender & & $\mathbf{n}$ \\
$\quad$ Males & $15(37 \%)$ & $7(35 \%)$ \\
$\quad$ Females & $26(63 \%)$ & $13(65 \%)$ \\
STAT3 mutation & $37(90 \%)$ & $17(85 \%)$ \\
Neutropenia (ANC $<\mathbf{1 , 5 0 0 )}$ & $37(90 \%)$ & $18(90 \%)$ \\
Severe neutropenia (ANC < 500) & $17(41 \%)$ & $2(10 \%)$ \\
\hline
\end{tabular}

ANC, absolute neutrophil count.

Interestingly, we observed that STAT3 phosphorylation was still present but lower in those patients whose mutation was detected only by ARMS-PCR, suggesting that STAT3 activation levels were likely correlated to the dimension of clone interested by mutation (Figure 4B).

\section{Fas ligand expression}

Since secreted Fas ligand, which is increased in the serum of patients affected by T-LGL leukemia [20-22], has been hypothesized to play a role in inducing neutropenia in these patients [16], we evaluated Fas ligand expression in our series of patients subdivided according to their immunophenotype. By Real-Time PCR, we observed that Fas ligand transcriptional expression median level was higher in CD16+/CD56- CD8+ T-LGL leukemia patients as compared with the non-neutropenic patients belonging to the other immunophenotypes, both CD8+ T-LGL leukemia and CD4+ T-LGL leukemia (7.66 $\pm 0.87,2.45$ \pm 0.22 and $2.35 \pm 0.28$ arbitrary units, respectively; $P<$ 0.001 ; Figure 5A). The difference of Fas ligand production observed on transcriptional expression was also confirmed by ELISA measuring Fas ligand level in patients' plasma (CD16+/CD56- CD8+ T-LGL leukemia: $88.3 \pm 14.18$ pg/ $\mathrm{ml}$, the other CD8+ and CD4+ immunophenotypes: 16.08 $\pm 14.62 \mathrm{pg} / \mathrm{ml}, P<0.0001$; Figure 5B).

\section{Fas ligand regulation}

To evaluate whether the high level of Fas ligand transcription depends on STAT3 activation, we treated PBMCs of patients in culture with Stattic, the specific inhibitor of STAT3 activation [23]. We observed that both STAT3 phosphorylation (Western Blot analysis, Figure $6 \mathrm{~A}$, wild type STAT3 on the upper panel and mutated STAT3 on the lower panel) and Fas ligand transcription (Real-Time PCR analysis, Figure 6B) slightly decreased after 2 hours culture with Stattic $(15 \mu \mathrm{M})$ as compared to the untreated conditions. Consistently, when we triggered STAT3 phosphorylation (Figure 6A) with IL-6 (20 ng/ $\mathrm{ml})$ or IL-15 (20 ng/ml), key cytokines in LGL leukemia development [9, 24], after one hour culture we observed an increase of Fas ligand transcription levels (1.59- and 2.01-fold after IL-6 and IL-15, respectively, Figure 6B). To verify whether Fas ligand expression was modulated just by STAT3 activation, before ILs incubation we pre-treated patients' PBMC for 1 hour with Stattic. We observed that Stattic blocked IL-6 or IL-15 effects preventing STAT3 phosphorylation induction (Figure $6 \mathrm{~A}$ ) and the increase of Fas ligand transcription (Figure $6 \mathrm{~B})$, thus demonstrating that the induction of Fas ligand expression is mediated by P-STAT3. This mechanism was observed in each immunophenotypic category of LGL leukemia patients, in detail, both STAT3 mutated patients and wild type patients, presenting different P-STAT3 and Fas ligand levels, showed similar molecular responses to IL-6 or IL-15 activation and Stattic inhibition. Our data demonstrated that STAT3 activation is crucial in Fas ligand regulation and explained the high levels of Fas ligand in CD8+/CD16+/CD56- patients characterized by high STAT3 activation.

\section{$S T A T 5 b$ mutations}

According to a recent report [13] showing high incidence of STAT5b mutations in CD4+ T-LGL leukemia, we evaluated also the presence of $S T A T 5 b$ mutations in all our cohort of study.

In our cohort, STAT5b mutations, represented by $\mathrm{N} 642 \mathrm{H}(n=2), \mathrm{Y} 665 \mathrm{~F}(n=2)$ and Q706L $(n=1)$, were found in 5 out of 101 patients (5\%). Interestingly, STAT5b mutations were present only in CD4+ T-LGL leukemia patients, confirming the data reported by Andersson et al [13], although with a lower incidence of $15.2 \%$ in CD4+ T-LGL leukemia (5 out of 33 patients) compared to $55 \%$ frequency reported by others, likely because of 


\section{A Fas ligand expression}

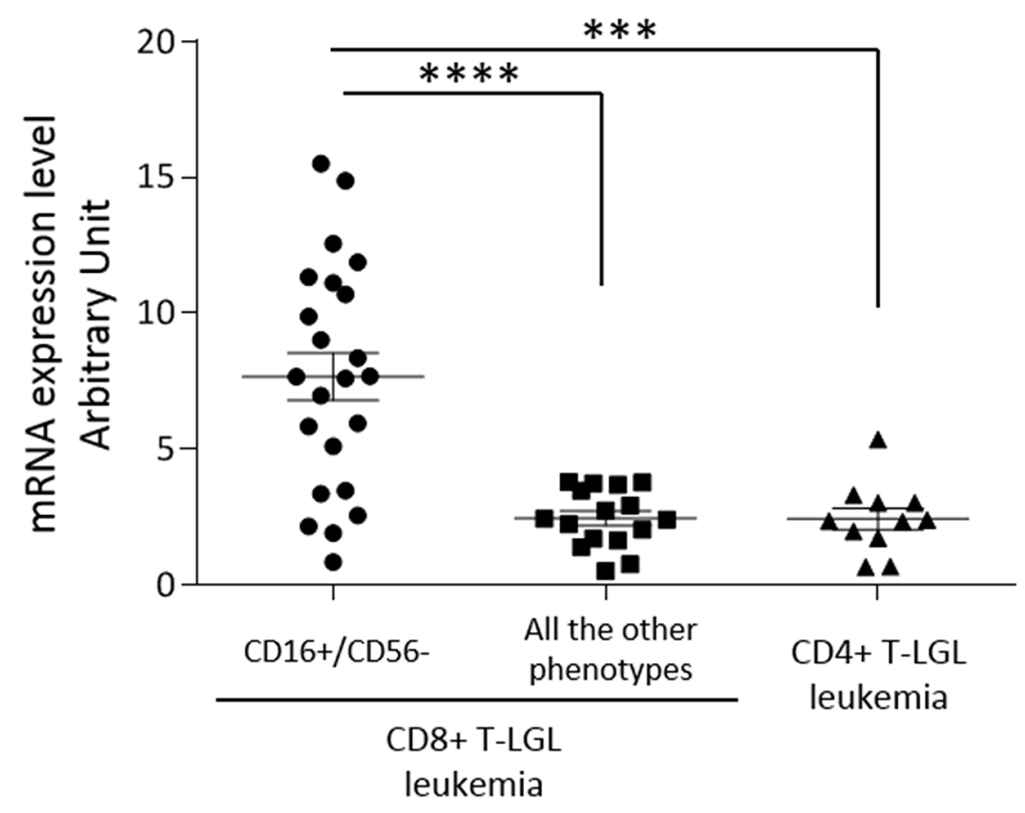

B Fas ligand level in plasma

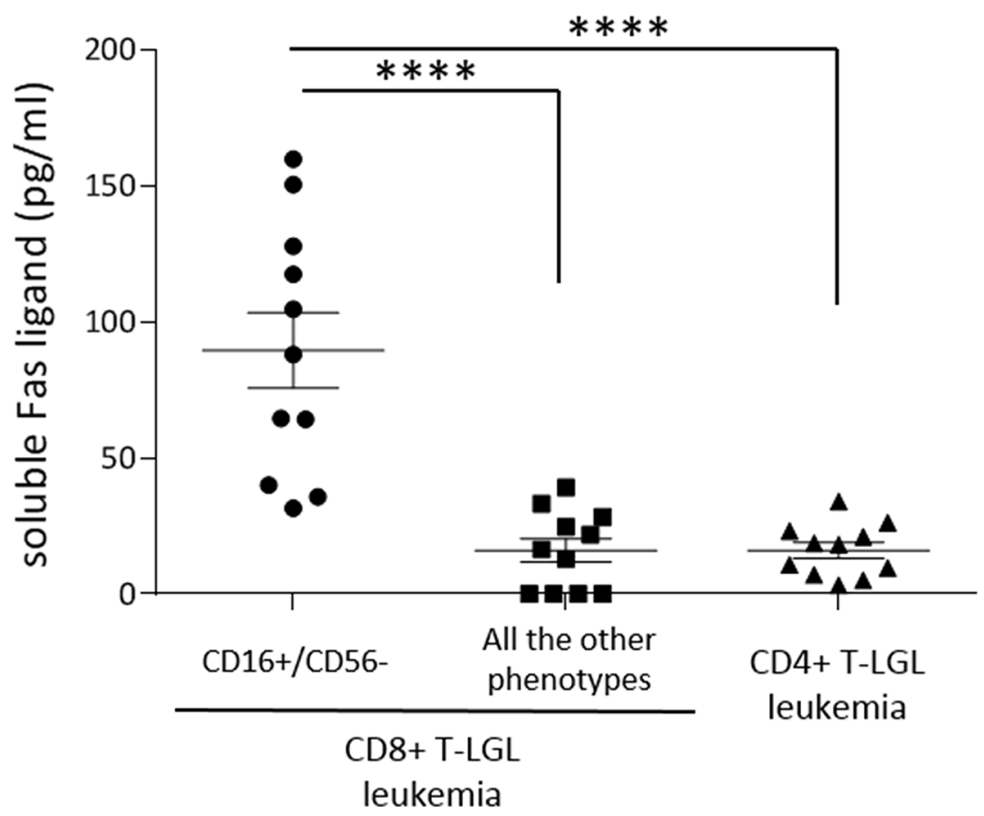

Figure 5: Fas ligand expression. Dot plots report (A) mRNA transcription levels and (B) plasma levels of secreted Fas ligand of T-LGL leukemia patients subdivided according to their immunophenotypes, CD8+ T-LGL leukemia (distinguished in patients with CD16+/CD56phenotype and those with all the other phenotypes) and CD4+ T-LGL leukemia. The means and SEM are reported. The expression level observed in the group of CD8+ T-LGL leukemia CD16+/CD56- subset is higher as compared with the two other groups $(* * * P<0.001$, $* * * * P<0.0001$, using one-way Anova and Tukey's multiple comparison test). 
A

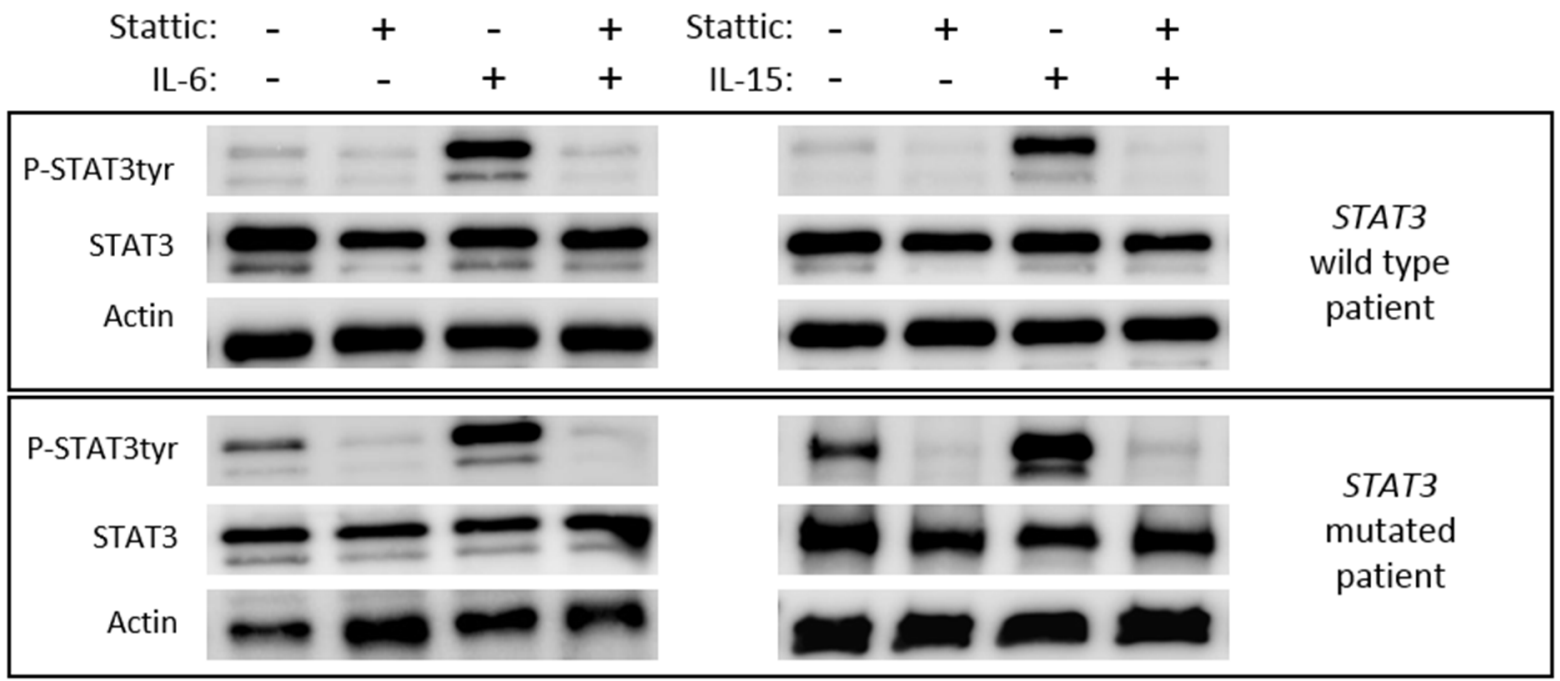

B

Fas ligand expression

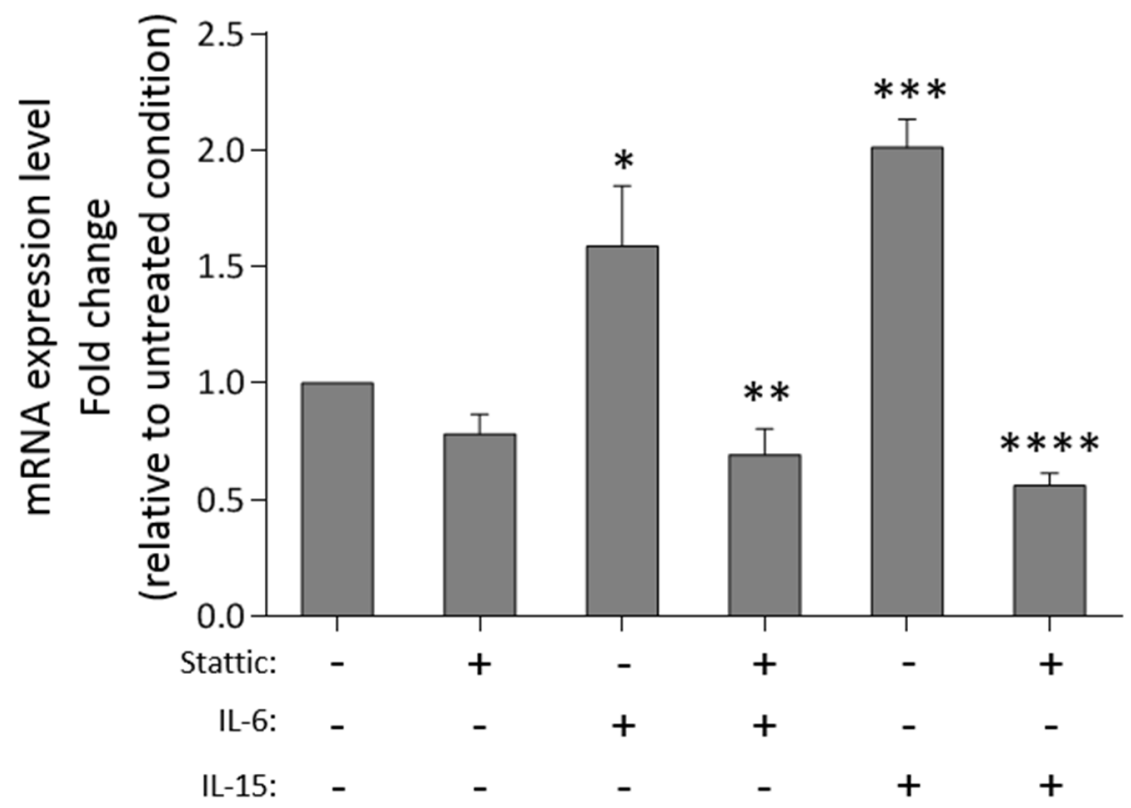

Figure 6: Fas ligand modulation after STAT3 activation/inhibition. Western blot analysis and Real-Time PCR results of patients' PBMCs after culture in the following different conditions: untreated condition (UT) for 2 hours; with Stattic (15 $\mu \mathrm{M})$ for 2 hours; stimulated by IL-6 $(20 \mathrm{ng} / \mathrm{ml})$ or IL-15 $(20 \mathrm{ng} / \mathrm{ml})$ for 1 hour; pretreated for 1 hour with Stattic and then stimulated by IL-6 or IL-15 for 1 hour. (A) STAT3 expression and tyrosine phosphorylation in whole-cell extracts were analyzed. Actin expression is shown as gel loading control. Upper panels show a representative STAT3 wild type sample, lower panels a representative STAT3 mutated sample. Data from two representatives out of six independent experiments are shown. (B) The histogram reports the average fold change of Fas ligand mRNA of patients' PBMCs. All the values were settled on untreated condition set at 1.0. Data are represented as mean \pm SEM of six independent experiments. $* P<0.05$ vs. UT; $* * P<0.01$ vs. IL- 6 condition; $* * * P<0.001$ vs. UT; $* * * * P<0.0001$ vs. IL- 15 condition, using one-way Anova and Tukey's multiple comparison test. 
the different number of patients studied (33 versus 11). Consistent with all CD4+ T-LGL leukemia patients, $S T A T 5 b$ mutated cases did not show any symptomatic clinical manifestation.

At variance to STAT3 mutated patient, analyzing CD16, CD56, CD57 cell markers no specific immunophenotype distinguishing STAT5b mutated from wild type patients was evidenced among CD4+ T-LGL leukemia. In fact, all CD4+ patients showed only two immunophenotypes, $78.8 \%$ characterized by the CD16-/ CD56+/CD57+ pattern and $21.2 \%$ characterized by the CD16+/CD56+/CD57+ pattern. STAT5b mutated patients were all equipped with the most frequent CD16-/CD56+/ CD57+ immunophenotype. Vbeta 13.1 prevalence observed in CD4+ T-LGL leukemia was similarly found in $S T A T 5 b$ mutated patients $(2 / 5,40 \%)$.

\section{DISCUSSION}

In a large cohort of T-LGL leukemia patients we showed that the CD8+/CD16+/CD56- LGL phenotype correlates with a specific subset of T-LGL leukemia patients, characterized by STAT3 mutations and neutropenia. To get insights into the molecular bases of neutropenia in these patients, we demonstrated a link between STAT3 phosphorylation and Fas ligand transcription levels. Supporting the correlation between mutational pattern and leukemic LGL phenotype, STAT5b mutations were specifically found only in CD4+ T-LGL leukemia.

At present STAT mutations are the most distinctive genetic lesions described in this disease and represent a molecular marker with high specificity [25], although not exclusive [26-28] of this lymphoproliferative disorder. At variance with other hematological conditions (i.e., Hairy Cell Leukemia, Waldenstrom Macroglobulinemia) $[29,30]$, the clinical significance of STAT mutations in patients with T-LGL leukemia has not been yet clarified. Some literature data correlated the presence of STAT3 mutations with neutropenia $[10,14]$, but these data were not confirmed by other authors $[11,31]$, moreover this correlation was not yet specifically evaluated in comparable series of cases. At variance $S T A T 5 b$ mutations have been reported to have a different clinical impact depending on which subset they were found, having been described to be associated to CD4+ T-LGL leukemia, characterized by indolent disease, [13] but having also been found in two cases of the rare aggressive form of LGL leukemia disorder (one with NK and the other with T type) [12].

Showing a close relationship between STAT3 genetic lesion and the presence of neutropenia, our results contribute to clarify the link between STAT3 mutations and symptomatic disease and, for the first time in a large series of patients, demonstrate an association between a specific immunophenotype and discrete biological and clinical features. Moreover, our results provide further evidence of the correlation between STAT5b mutations and CD4+ T-LGL leukemia, recently described in a small cohort of cases $(n=11)$ and here confirmed in a larger cohort of patients $(n=33)$ even if with a lower incidence $(15.2 \%$ vs $55 \%$ reported [13]).

Interestingly, our results indicate that $\mathrm{CD} 8+$ and CD4+ T-LGL leukemia present different biologic background. In fact, STAT3 and STAT5b mutations represent an exclusive marker of CD8+ T-LGL leukemia and CD4+ T-LGL leukemia, respectively. Among the different CD8+ T-LGL leukemia immunophenotypic combinations, patients characterized by CD16+/CD56proliferating cells showed the highest frequency of STAT3 mutations. Only 4 out of 41 cases within this specific immunophenotypic subgroup were devoid of STAT3 mutations. It might be speculated that STAT3 mutations could indeed occur in these patients, but present in only a very low subset of LGL clone (ARMS is currently available only for the two main mutations, Y640F and D661Y) or likely located at sites not yet screened. Supporting this last hypothesis, in our study group we identified also mutations that are not already reported, located on exon 21 , where the main mutations have been found, more precisely in-frame deletions/ insertions (Figure 1). Furthermore, a recent paper described mutations that, even if located outside the $\mathrm{SH} 2$ domain, were able to induce a gain of function of STAT3 [32]. On this basis we can speculate that mutation analysis might actually underestimate the real incidence of STAT3 genetic lesions. To strength the significance of our results, they were validated on a cohort of 20 patients coming from France, confirming that the CD8+/CD16+/ CD56- immunophenotype might have a prognostic value in T-LGL leukemia patients, thus contributing to the identification of patients most at risk of neutropenia, for whom STAT3 mutation analysis is highly recommended. This validation, based on retrospective data, takes into account only patients with the phenotype of interest within the French cohort. A larger validation cohort including all the possible phenotypes would have been preferred, but it would have been possible only by performing a multicentre study unifying the criteria of evaluation of all the different variables. Concerning this point, according to our experience, we emphasize that a correct immunophenotype characterization must be performed not only in whole blood but also on purified PBMCs, since total blood CD16 population is frequently underestimated or even unscreened because of the anti-CD16 specific antibody binding to granulocytes.

In terms of the mechanisms accounting for neutropenia in CD8+/CD16+/CD56- phenotypic subset characterized by STAT3 mutations, our data suggest a pathogenetic link between STAT3 activation and the development of neutropenia. According to the finding that mutations confer STAT3 activation [10], we observed 
higher P-STAT3 in mutated patients. Interestingly, high levels of P-STAT3 tyr705 were demonstrated mostly on CD8+/CD16+/CD56- LGL patients and also in the 4 patients of this subset lacking STAT3 mutations, suggesting that a strong STAT3 activation might represent a specific hallmark of this immunophenotypic subset. Consistently, STAT3 activation in other immunophenotypic subsets was lower. Liu and co-workers [16] showed that neutropenia in LGL leukemia patients was consequent to the high level of circulating Fas ligand produced by LGLs, since neutrophils undergo apoptosis through Fas triggering. Here we provide evidence that Fas ligand expression was specifically higher in patients characterized by CD8+/CD16+/CD56- phenotype as compared to other subsets. We also demonstrated that this higher Fas ligand transcription was mediated by STAT3 activation. In fact, we found that Stattic, a specific inhibitor of STAT3 phosphorylation, was able to reduce Fas ligand transcription levels. Furthermore, inducing STAT3 phosphorylation with IL-6 or IL-15 we observed an increase of Fas ligand transcription that was prevented through Stattic exposure. These results might explain why Fas ligand was found to be more expressed in CD8+/ CD16+/CD56- patients, who are specifically characterized by higher level of P-STAT3.

Confirming the well-known heterogeneity of T-LGL leukemia, this study, performed in a large cohort of patients, provides evidence for a link between biological markers (phenotype, STAT3 mutations and activation, Fas ligand production) and neutropenia. Our results also contribute to clearly separate CD4+ from CD8+ T-LGL leukemia, the first group being characterized by very low frequency of neutropenia and lack of STAT3 mutations.

In conclusion, our data emphasize the relevance of flow cytometry and STAT3 mutation analysis in order to gain information on clinical course and biologic features of disease, thus correctly addressing the management of each patient. Moreover, our results show that already at diagnosis flow cytometry evaluation might represent a valuable tool to identify patients who need further investigation.

\section{MATERIALS AND METHODS}

\section{Study patients}

The pilot study population consisted of 101 patients affected by T-LGL leukemia from Hematology Unit of Padua (Italy). Additional 20 patients, representing a validation cohort, were recruited from Hematology Unit of Rennes (France). All 121 patients met the 2008 WHO (World Health Organization) criteria for T-LGL leukemia diagnosis with LGL expansion $(>500 / \mu 1)$ persisting for at least 6 months; clonality was demonstrated by molecular analysis of T-cell receptor (TCR) gene rearrangement in all cases. Patients characteristics were evaluated, including the presence of cytopenia, association with autoimmune disease, secondary neoplasia and treatment requirement. Median follow-up was 9 years, ranging from 3 to 16 years. Neutropenia, particularly severe neutropenia, was confirmed in repeated ANC performed every 1-6 months depending on individual clinical characteristics during the follow-up.

All samples were recruited at diagnosis and during the disease follow-up. This study was performed according to the Helsinki Declaration and patients gave written informed consensus prior to inclusion in the study. The study and blood sample collection were approved by Ethic Committee for Clinical Trial of Padua.

\section{Flow cytometry analysis}

The frequency of LGLs positive for the characteristic antigens was assessed by flow cytometry analysis using direct or indirect immunofluorescence assay combining up to 6 fluorescences. Briefly, cells were stained with the appropriate mAbs either unlabeled or labeled with different fluorochromes; staining with unlabeled $\mathrm{mAb}$ was followed by conjugated isotypespecific goat anti-mouse secondary reagent (Southern Biotechnology, Birmingham, AL, or Caltag, Burlingame, CA). The commercially available FITC-, phycoerythrin (PE)-, PeCy5-conjugated, PeCy7-conjugated, APCconjugated and APC-Cy7-conjugated mouse monoclonal antibodies (mAbs) used included: anti-CD3 (SK7), antiCD4 (RPA-T4), anti-CD8 (RPA-T8), anti-CD16 (3G8), anti-CD56 (NCAM16.2) and anti-CD57 (NK-1) from Becton Dickinson (Sunnyvale, CA, USA).

Cells were scored using a FACSCanto analyzer (BD Biosciences, San Jose CA) and data processed by the BD FACSDiva software program (BD Biosciences). The investigation for LGL surface markers (CD3, CD4, CD8, CD16, CD56, CD57) was performed on peripheral blood and repeated also on fresh PBMCs, since in total blood CD16 was frequently underestimated or even unscreened because the specific antibody anti-CD16 was sequestered by granulocytes.

\section{Isolation of LGLs from patients with T-LGL leukemia}

PBMCs were obtained by Ficoll-Hypaque (Sigma Aldrich) gradient centrifugation. Patients' T-LGLs were obtained using magnetic separations over columns (MACS; Miltenyi Biotec, Auburn, CA) with magnetic Micro-Beads coated with monoclonal anti-human CD57, CD56 or CD16 antibodies (Miltenyi Biotec). Alternatively, LGLs were obtained from PBMCs by the FACSAria cell sorter (BD Biosciences) making the selection according to CD57, CD56 or CD16 antigen expression. Sorted populations were analyzed for purity and viability (both $>$ $95 \%$ ). In preliminary experiments we ruled out that cells 
obtained by FACSAria sorting or by magnetic MicroBeads were functionally different.

\section{Screening of STAT mutations}

For the screening of STAT3 and STAT5 $b$ mutations we used the set of primers reported by Koskela et al [10] and by Rajala et al [12], respectively, to amplify the hot spot regions for mutations (exon 21 for STAT3 and exons 16-18 for $S T A T 5 b$ ). DNA from purified LGLs and from the remaining autologous PBMCs was separately analyzed. DNA was extracted from 1-20 x $10^{6}$ cells using the Puregene Cell Kit Plus (Qiagen) and then sequenced using dye terminator technology and an ABI 3130 sequencer (Applied Biosystems). The presence of D661Y and Y640F STAT3 mutations undetectable by direct sequencing, because of the limited sensitivity of the method (reaching $25 \%$ of positive cells, as previously established) [9], was also analyzed by a DNA tetraprimer amplification refractory mutation system assay (ARMSPCR), as reported by Jerez et al [11].

\section{Western blot analysis}

Cells (2.5 x $10^{5}$ for each assay) were boiled for 7 minutes in Laemmli sample buffer. Samples were then subjected to sodium dodecyl sulfate/polyacrylamide gel electrophoresis (acrylamide 10\% gels), transferred to PVDF membranes, immunostained with the specific antibodies and at the end with monoclonal anti- $\beta$-actin and revealed using an enhanced chemiluminescent detection system (Thermo Scientific; Waltham, MA, USA). The blots were acquired with the ImageQuant LAS 500 and analyzed by ImageQuant TL v8.1 software (GE Healthcare; Buckingamshire, UK). For Western blot analysis the following primary or secondary antibodies were used: polyclonal rabbit anti-STAT3 (79D7) and anti-phospho STAT3 Tyrosine 705 (D3A7) antibodies from Cell Signaling (Danvers, MA); monoclonal mouse anti- $\beta$-actin (AC-15) antibody from Sigma Aldrich (St. Louis, MO); horseradish peroxidise-conjugated antirabbit and anti-mouse immunoglobulins from PerkinElmer (Waltham, MA, USA).

\section{Real-Time PCR}

Total cellular RNA was extracted from cells using RNeasy Mini Kit (Qiagen, Hilden, Germany), according to the manufacturer's protocol and treated with DNase (Qiagen). Complementary DNA was generated from $1 \mu \mathrm{g}$ total RNA using oligo-dT primer and the AMV reverse transcriptase (Promega, Madison, WI, USA). Real-Time polymerase chain reaction was carried out in an ABI Prism 7000 sequence detection system (Applied Biosystems, Foster City, CA). SYBR Green PCR Master Mix was purchased from Roche. The primers used are:
Fas ligand, forward 5'-GCTGCCACCCCTGAAGAA-3', reverse 5'-ATGAAAAACATCACAAGGAGACACA-3'; GAPDH (glyceraldehyde-3-phosphate dehydrogenase), forward 5'-AATGGAAATCCCATCACCATCT-3', reverse 5'-CGCCCCACTTGATTTTGG-3'. The primers were designed by Primer Express 3.0 (Applied Biosystems). The results were obtained with Delta Delta $\mathrm{Ct}$ analysis. The relative amounts of mRNA were normalized for GAPDH expression.

\section{Cell culture}

PBMCs from patients were cultured at $2 \times 10^{6}$ cells/ $\mathrm{ml}$ in RPMI-1640 (EuroClone) medium, supplemented with $10 \%$ fetal calf serum, $100 \mathrm{U}$ of penicillin and $100 \mu \mathrm{g}$ streptomycin (EuroClone) per $\mathrm{ml}$ and grown in $5 \% \mathrm{CO}_{2}$ at $37^{\circ} \mathrm{C}$. We left cell culture untreated for 24 hours, then we treated with Stattic (15 $\mu \mathrm{M}$, Selleckchem) for one hour, specifically inhibiting STAT3 phosphorylation, dimerization and nuclear translocation [23], prior to stimulation by IL-6 (20 ng/ml; Sigma Aldrich) or IL-15 (20 ng/ml; Peprotech) for one hour.

\section{Enzyme-linked immunosorbent assay (ELISA)}

Levels of secreted Fas ligand in plasma were determined by ELISA kit (RayBiotech, Georgia, USA), following the manufacturer's recommendations. A standard curve was generated using seven solutions of recombinant human Fas ligand with known concentrations $(1,000,333.3,111.1,37.04,12.35,4.12$, and $1.37 \mathrm{pg} / \mathrm{ml})$. Briefly, $100 \mu \mathrm{l}$ of each standard or sample, in duplicate, was added to each well of a 96-well ELISA plate and incubated 2.5 hours at room temperature. After four consecutive washes $(4 \times 300 \mu 1$ with Wash Buffer $), 100 \mu \mathrm{l}$ of biotin antibody was added to each well and incubated at room temperature, for 1 hour. After a second washing step $(4 \times 300 \mu \mathrm{l}), 100 \mu \mathrm{l}$ of Streptavidin solution was added and incubated at room temperature for 45 minutes. After a third washing step $(4 \times 300 \mu \mathrm{l}), 100 \mu \mathrm{l}$ of tetramethylbenzidine (TMB) substrate reagent was added to each well and incubated for 30 minutes at room temperature in the dark. The reaction was interrupted by adding $50 \mu \mathrm{l}$ of stop solution per well and absorbance was immediately read at $450 \mathrm{~nm}$ by a microplate reader platform (Victor Multilabel plate reader, PerkinElmer).

\section{Statistics}

Data are expressed as mean plus or minus the standard error mean (SEM) and statistical analysis was performed by one-way Anova followed by Tukey's multiple comparison test. Comparisons of proportions and ranks of variables between groups were performed by $\chi^{2}$ test. All the analyses were undertaken using GraphPad Prism 6. A value of $P<0.05$ was accepted as significant. 


\section{Abbreviations}

$\mathrm{LGL}=$ large granular lymphocyte, $\mathrm{KIR}=$ killer immunoglobulin-like receptor, $\mathrm{AICD}=$ activation induced cell death, ARMS = amplification refractory mutation system, STAT3 = signaling transducer and activator of transcription 3, SOCS3 = suppressor of cytokine signaling $3, \mathrm{PBMC}=$ peripheral blood mononuclear cells, $\mathrm{ANC}=$ absolute neutrophil count, RA = rheumatoid arthritis, MTX $=$ methotrexate, $\mathrm{WHO}=$ World Health Organization, $\mathrm{TCR}$ $=\mathrm{T}$-cell receptor, ELISA $=$ Enzyme-linked immunosorbent assay, $\mathrm{UT}=$ untreated condition

\section{Author contributions}

AT and GB designed the research, performed some of the in vitro research, analyzed data and wrote the manuscript. GC, CE and ML performed some of the in vitro research. TL, AM, MR and CP provided the data about the validation cohort of patients from Rennes, France. CG performed some of the in vitro research and participated in analysis of data. AC, EB and ST performed flow cytometer studies. EP performed Sanger sequencing. TB and EDM provided patient samples and patient data. MF, FP and LT contributed to analyze data. GS provided funding, participated to the analysis of data and critically reviewed and edited the manuscript. RZ designed the study, analyzed data, wrote the manuscript and supervised the study.

\section{CONFLICTS OF INTEREST}

The authors declare no competing financial interests.

\section{FUNDING}

This work was supported by Associazione Italiana per la Ricerca sul Cancro (AIRC, IG-15286), Cariparo, Cariverona, Ministero dell'Università e della Ricerca Scientifica e Tecnologica (MURST), Lions Club International and Associazione Italiana contro le Leucemie, Linfomi e Mieloma (AIL).

\section{REFERENCES}

1. Semenzato G, Zambello R, Starkebaum G, Oshimi K, Loughran TP Jr. The lymphoproliferative disease of granular lymphocytes: updated criteria for diagnosis. Blood. 1997; 89:256-260.

2. Wlodarski MW, Schade AE, Maciejewski JP. T-large granular lymphocyte leukemia: current molecular concepts. Hematology. 2006; 11:245-256.

3. Loughran TP Jr. Clonal diseases of large granular lymphocytes. Blood. 1993; 82:1-14.

4. Zambello R, Semenzato G. Large granular lymphocytosis. Haematologica. 1998; 83:936-942.
5. Baesso I, Pavan L, Boscaro E, Miorin M, Facco M, Trentin L, Agostini C, Zambello R, Semenzato G. T-cell type lymphoproliferative disease of granular lymphocytes (LDGL) is equipped with a phenotypic pattern typical of effector cytotoxic cells. Leuk Res. 2007; 31:371-377.

6. Garrido P, Ruiz-Cabello F, Barcena P, Sandberg Y, Canton J, Lima M, Balanzategui A, Gonzalez M, Lopez-Nevot MA, Langerak AW, Garcia-Montero AC, Almeida J, Orfao A. Monoclonal TCR-Vbeta13.1+/CD4+/NKa+/CD8-/+dim T-LGL lymphocytosis: evidence for an antigen-driven chronic T-cell stimulation origin. Blood. 2007; 109:4890-4898.

7. Leblanc F, Zhang D, Liu X, Loughran TP. Large granular lymphocyte leukemia: from dysregulated pathways to therapeutic targets. Future Oncol. 2012; 8:787-801.

8. Epling-Burnette PK, Liu JH, Catlett-Falcone R, Turkson J, Oshiro M, Kothapalli R, Li Y, Wang JM, Yang-Yen HF, Karras J, Jove R, Loughran TP Jr. Inhibition of STAT3 signaling leads to apoptosis of leukemic large granular lymphocytes and decreased Mcl-1 expression. J Clin Invest. 2001; 107:351-362.

9. Teramo A, Gattazzo C, Passeri F, Lico A, Tasca G, Cabrelle A, Martini V, Frezzato F, Trimarco V, Ave E, Boscaro E, Piazza F, Facco M, et al. Intrinsic and extrinsic mechanisms contribute to maintain the JAK/STAT pathway aberrantly activated in T-type large granular lymphocyte leukemia. Blood. 2013; 121:3843-3854.

10. Koskela HL, Eldfors S, Ellonen P, van Adrichem AJ, Kuusanmäki H, Andersson EI, Lagström S, Clemente MJ, Olson T, Jalkanen SE, Majumder MM, Almusa $\mathrm{H}$, Edgren $\mathrm{H}$, et al. Somatic STAT3 mutations in large granular lymphocytic leukemia. N Engl J Med. 2012; 366:1905-1913.

11. Jerez A, Clemente MJ, Makishima H, Koskela H, Leblanc F, Peng Ng K, Olson T, Przychodzen B, Afable M, GomezSegui I, Guinta K, Durkin L, Hsi ED, et al. STAT3 mutations unify the pathogenesis of chronic lymphoproliferative disorders of NK cells and T-cell large granular lymphocyte leukemia. Blood. 2012; 120:3048-3057.

12. Rajala HL, Eldfors S, Kuusanmaki H, van Adrichem AJ, Olson T, Lagstrom S, Andersson EI, Jerez A, Clemente MJ, Yan Y, Zhang D, Awwad A, Ellonen P, et al. Discovery of somatic STAT5b mutations in large granular lymphocytic leukemia. Blood. 2013; 121:4541-4550.

13. Andersson EI, Tanahashi T, Sekiguchi N, Gasparini VR, Bortoluzzi S, Kawakami T, Matsuda K, Mitsui T, Eldfors S, Coppe A, Binatti A, Lagstrom S, Ellonen P, et al. High incidence of activating STAT5B mutations in CD4-positive T-cell large granular lymphocyte leukemia. Blood. 2016; 128:2465-2468.

14. Qiu ZY, Fan L, Wang L, Qiao C, Wu YJ, Zhou JF, Xu W, Li JY. STAT3 mutations are frequent in T-cell large granular lymphocytic leukemia with pure red cell aplasia. J Hematol Oncol. 2013; 6:82.

15. Pontikoglou C, Kalpadakis C, Papadaki HA. Pathophysiologic mechanisms and management of 
neutropenia associated with large granular lymphocytic leukemia. Expert Rev Hematol. 2011; 4:317-328.

16. Liu JH, Wei S, Lamy T, Epling-Burnette PK, Starkebaum G, Djeu JY, Loughran TP. Chronic neutropenia mediated by fas ligand. Blood. 2000; 95:3219-3222.

17. Lamy T, Loughran TP Jr. Clinical features of large granular lymphocyte leukemia. Semin Hematol. 2003; 40:185-195.

18. Sokol L, Loughran TP Jr. Large granular lymphocyte leukemia. Oncologist. 2006; 11:263-273.

19. Loughran TP Jr, Zickl L, Olson TL, Wang V, Zhang D, Rajala HL, Hasanali Z, Bennett JM, Lazarus HM, Litzow MR, Evens AM, Mustjoki S, Tallman MS. Immunosuppressive therapy of LGL leukemia: prospective multicenter phase II study by the Eastern Cooperative Oncology Group (E5998). Leukemia. 2015; 29:886-894.

20. Tanaka M, Suda T, Haze K, Nakamura N, Sato K, Kimura F, Motoyoshi K, Mizuki M, Tagawa S, Ohga S, Hatake K, Drummond AH, Nagata S. Fas ligand in human serum. Nat Med. 1996; 2:317-322.

21. Perzova R, Loughran TP Jr. Constitutive expression of Fas ligand in large granular lymphocyte leukaemia. Br J Haematol. 1997; 97:123-126.

22. Lamy T, Liu JH, Landowski TH, Dalton WS, Loughran TP Jr. Dysregulation of CD95/CD95 ligand-apoptotic pathway in CD3(+) large granular lymphocyte leukemia. Blood. 1998; 92:4771-4777.

23. Schust J, Sperl B, Hollis A, Mayer TU, Berg T. Stattic: a small-molecule inhibitor of STAT3 activation and dimerization. Chem Biol. 2006; 13:1235-1242.

24. Zambello R, Facco M, Trentin L, Sancetta R, Tassinari C, Perin A, Milani A, Pizzolo G, Rodeghiero F, Agostini C, Meazza R, Ferrini S, Semenzato G. Interleukin-15 triggers the proliferation and cytotoxicity of granular lymphocytes in patients with lymphoproliferative disease of granular lymphocytes. Blood. 1997; 89:201-211.

25. Rajala HL, Olson T, Clemente MJ, Lagstrom S, Ellonen P, Lundan T, Hamm DE, Zaman SA, Lopez Marti JM, Andersson EI, Jerez A, Porkka K, Maciejewski JP, et al.
The analysis of clonal diversity and therapy responses using STAT3 mutations as a molecular marker in large granular lymphocytic leukemia. Haematologica. 2015; 100:91-99.

26. Crescenzo R, Abate F, Lasorsa E, Tabbo F, Gaudiano M, Chiesa N, Di Giacomo F, Spaccarotella E, Barbarossa L, Ercole E, Todaro M, Boi M, Acquaviva A, et al. Convergent mutations and kinase fusions lead to oncogenic STAT3 activation in anaplastic large cell lymphoma. Cancer Cell. 2015; 27:516-532.

27. Ohgami RS, Ma L, Merker JD, Martinez B, Zehnder JL, Arber DA. STAT3 mutations are frequent in CD30+ T-cell lymphomas and T-cell large granular lymphocytic leukemia. Leukemia. 2013; 27:2244-2247.

28. Ohgami RS, Ma L, Monabati A, Zehnder JL, Arber DA. STAT3 mutations are present in aggressive B-cell lymphomas including a subset of diffuse large B-cell lymphomas with CD30 expression. Haematologica. 2014; 99:e105-107.

29. Tiacci E, Trifonov V, Schiavoni G, Holmes A, Kern W, Martelli MP, Pucciarini A, Bigerna B, Pacini R, Wells VA, Sportoletti P, Pettirossi V, Mannucci R, et al. BRAF mutations in hairy-cell leukemia. N Engl J Med. 2011; 364:2305-2315.

30. Treon SP, Xu L, Yang G, Zhou Y, Liu X, Cao Y, Sheehy P, Manning RJ, Patterson CJ, Tripsas C, Arcaini L, Pinkus GS, Rodig SJ, et al. MYD88 L265P somatic mutation in Waldenstrom's macroglobulinemia. N Engl J Med. 2012; 367:826-833.

31. Ishida F, Matsuda K, Sekiguchi N, Makishima H, Taira C, Momose K, Nishina S, Senoo N, Sakai H, Ito T, Kwong YL. STAT3 gene mutations and their association with pure red cell aplasia in large granular lymphocyte leukemia. Cancer Sci. 2014; 105:342-346.

32. Andersson E, Kuusanmaki H, Bortoluzzi S, Lagstrom S, Parsons A, Rajala H, van Adrichem A, Eldfors S, Olson T, Clemente MJ, Laasonen A, Ellonen P, Heckman C, et al. Activating somatic mutations outside the SH2-domain of STAT3 in LGL leukemia. Leukemia. 2016; 30:1204-1208. 